\title{
A STUDY OF PULSE-ECHO IMAGE FORMATION USING NON-QUADRATIC REGULARIZATION WITH SPECKLE-BASED IMAGES
}

\author{
R. Lavarello, F. Kamalabadi, W.D. O’Brien, Jr. \\ Bioacoustics Research Laboratory, Coordinated Science Laboratory, Department of \\ Electrical and Computer Engineering, University of Illinois at Urbana-Champaign, 405 North \\ Mathews, Urbana, IL 61801 USA
}

Abstract:

Objective: B-mode imaging is a standard way of presenting results when pulse-echo data acquisition is used. Inverse approaches have been explored to a limited extent. The goals of this study are to explore the feasibility and effectiveness of regularization by: (a) evaluating the quality of the reconstruction of speckle-based images as a function of imaging parameters (i.e., bandwidth of the transducer, f/number, and distance between region of interest (ROI) and focal region), and (b) comparing the reconstructed images with those obtained by conventional imaging techniques (e.g., B-mode and synthetic aperture focusing techniques (SAFT)). Methodology: Twodimensional pulse-echo data from a single-element focused transducer with center frequency of $6 \mathrm{MHz}$ and focal distance of $19 \mathrm{~mm}$ were simulated using fractional bandwidths of $33 \%, 50 \%$ and $100 \%$, f/numbers of 1,2 and 3 , and distances between ROI and focus of $0,2.5$ and $5 \mathrm{~mm}$. A minimum scatterer density of 4 scatterers per resolution cell was used. The images were reconstructed using non-quadratic regularization. Because the desired image was known, the normalized mean squared error (MSE) was used as a quantitative indicator of reconstruction performance. The reconstructed images were also evaluated by visual inspection. Results: The best reconstructions were achieved when the ROI was at the focus, the bandwidth was $100 \%$ and the $\mathrm{f} /$ number was 1 , for which the MSE was $25 \%$ for a $20-\mathrm{dB}$ signal-to-noise ratio (SNR). The MSE increased as the bandwidth decreased, the $\mathrm{f} /$ number increased, and the distance between ROI and focus increased. The bandwidth was the most sensitive parameter, increasing the MSE up to $86 \%$ for a SNR of $20 \mathrm{~dB}$. For all cases, parameter selection techniques such as the L-curve and generalized cross-validation (GCV) gave a close 
approximation to the image with the best visual appearance. Conclusion: For speckle-based images the inversion using regularization gave better results than conventional techniques for high SNR. A lower bound of $20 \mathrm{~dB}$ was found for this study. Below this threshold contrast information and most of the image features were lost during the reconstruction process in order to control the distortion due to the noise. Further work will be required to improve the stability of the forward model and to use more appropriate a priori model information in order to use this technique in a more realistic scenario including the effect of modeling errors

Key words: Inverse problems; non quadratic regularization; speckle-based images

\section{INTRODUCTION}

B-mode, synthetic aperture focusing techniques (SAFT) and array imaging have been studied for a long time and their limitations are well documented ${ }^{1}$. Such techniques suffer from spatial resolution limitations due to the finite size of the resolution cell of the imaging system, i.e., the area (or volume) that corresponds to the smallest resolvable detail, and contrast resolution limitations due to the presence of sidelobes. Because ultrasonic imaging is coherent, when there are many scatterers randomly distributed in a resolution cell, their reflections may interfere constructively or destructively depending on their relative position to the transducer generating spots of brightness and darkness in the image. For focused systems, these spots tend to have a size on the order of the focal region and the resulting image will have a granular appearance resulting in speckle-based images ${ }^{2}$. Speckle is very common in ultrasound medical imaging because body tissue is a semitransparent media. If the point spread function has a wide mainlobe or high-level sidelobes, the spots generated by the speckle can mask regions of low contrast.

Inverse problem approaches ${ }^{3}$ and regularization techniques, such as truncated singular value decomposition (TSVD) ${ }^{4}$, total variation ${ }^{5}$, total least squares $^{6}$ and others ${ }^{7}$, have already been used in acoustical imaging and their effectiveness have been explored to a limited extent. It is of special importance to compare the performance of this class of techniques with that which can be obtained using conventional acoustic imaging methods. Speckle based images are proposed as targets of reconstruction because of the limitations of conventional techniques in dealing with these type of images. The goals of this study are to explore the feasibility and effectiveness of regularization by: (a) evaluating the quality of the reconstruction of speckle-based images as a function of imaging parameters 
(i.e., bandwidth of the transducer, f/number, and distance between region of interest (ROI) and focal region), and (b) comparing the reconstructed images with those obtained by conventional imaging techniques (e.g., B-mode and SAFT).

\section{METHODOLOGY}

The ROI is a square region with axial (or beam) axis $\mathrm{x}$ and lateral (or scanning) axis $y$. The pulse-echo data are acquired at locations $\mathrm{u}_{\mathrm{L}}, \mathrm{L} \in[1, \mathrm{q}]$. The received signal is sampled at discrete times $\mathrm{t}_{\mathrm{k}}, \mathrm{k} \in[1, \mathrm{p}]$. The time signal recorded at the position $\mathrm{u}_{\mathrm{L}}$ is denoted by $\mathrm{g}_{\mathrm{u}_{\mathrm{L}}}(\mathrm{t})$. The ROI is also discretized, with the discrete values of $\mathrm{x}$ and $\mathrm{y}$ denoted by $\mathrm{x}_{\mathrm{i}}, \mathrm{i} \in[1, \mathrm{n}]$ and $y_{i}, i \in[1, m]$. The reflectance of the ROI will be assumed to be constant in each pixel and will be denoted by $\mathrm{r}_{\mathrm{x}_{\mathrm{i}}, \mathrm{y}_{\mathrm{j}}}$. The spatially-variant impulse response of the transducer, i.e., the signal that would be received if a point target of unitary amplitude were at the coordinates $(\hat{\mathrm{x}}, \hat{\mathrm{y}})$ relative to the transducer, will be denoted by $s_{\hat{x}, \hat{y}}(t)$.

Neglecting multiple scattering, the received pulse-echo data when the transducer is placed at the location $\mathrm{u}_{\mathrm{L}}$ will be the sum of the impulse responses of all the pixels inside the ROI scaled by the corresponding values of the reflectance. The two-dimensional sequences $\mathrm{g}\left(\mathrm{t}, \mathrm{u}_{\mathrm{L}}\right)$ and $\mathrm{r}\left(\mathrm{x}_{\mathrm{i}}, \mathrm{y}_{\mathrm{j}}\right)$ are stacked as vectors to form a pair of one-dimensional sequences in order to express the problem as a set of linear equations. With this reordering, the forward model can be written in matrix form as: 


$$
\left[\begin{array}{l}
{\left[\begin{array}{l}
s_{x_{1}, y_{1}-u_{1}}\left(t_{1}\right) \\
s_{x_{1}, y_{1}-u_{1}}\left(t_{2}\right) \\
\vdots \\
s_{x_{1}, y_{1}-u_{1}}\left(t_{p}\right)
\end{array}\right]\left[\begin{array}{l}
s_{x_{2}, y_{1}-u_{1}}\left(t_{1}\right) \\
s_{x_{2}, y_{1}-u_{1}}\left(t_{2}\right) \\
\vdots \\
s_{x_{2}, y_{1}-u_{1}}\left(t_{p}\right)
\end{array}\right] \cdots\left[\begin{array}{l}
s_{x_{n}, y_{m}-u_{1}}\left(t_{1}\right) \\
s_{x_{n}, y_{m}-u_{1}}\left(t_{2}\right) \\
\vdots \\
s_{x_{n}, y_{m}-u_{1}}\left(t_{p}\right)
\end{array}\right]} \\
{\left[\begin{array}{l}
{\left[\begin{array}{l}
s_{x_{1}, y_{1}-u_{q}}\left(t_{1}\right) \\
s_{x_{1}, y_{1}-u_{q}}\left(t_{2}\right) \\
\vdots \\
s_{x_{1}, y_{1}-u_{q}}\left(t_{p}\right)
\end{array}\right]\left[\begin{array}{l}
s_{x_{2}, y_{1}-u_{q}}\left(t_{1}\right) \\
s_{x_{2}, y_{1}-u_{q}}\left(t_{2}\right) \\
\vdots \\
s_{x_{2}, y_{1}-u_{q}}\left(t_{p}\right)
\end{array}\right] \ldots\left[\begin{array}{l}
s_{x_{n}, y_{m}-u_{q}}\left(t_{1}\right) \\
s_{x_{n}, y_{m}-u_{q}}\left(t_{2}\right) \\
\vdots \\
s_{x_{n}, y_{m}-u_{q}}\left(t_{p}\right)
\end{array}\right]}
\end{array}\right]\left[\begin{array}{l}
{\left[\begin{array}{l}
g_{u_{1}}\left(t_{1}\right) \\
g_{u_{1}}\left(t_{2}\right) \\
\vdots \\
r_{x_{1}, y_{1}} \\
r_{x_{2}, y_{1}} \\
r_{x_{3}, y_{1}} \\
\vdots \\
g_{u_{1}}\left(t_{p}\right)
\end{array}\right]=\left[\begin{array}{l}
\vdots \\
r_{x_{n}, y_{m}}
\end{array}\right]} \\
{\left[\begin{array}{l}
g_{u_{q}}\left(t_{1}\right) \\
g_{u_{q}}\left(t_{2}\right) \\
\vdots \\
g_{u_{q}}\left(t_{p}\right)
\end{array}\right]}
\end{array}\right]}
\end{array}\right.
$$

Because the forward model matrix $\mathrm{S}$ is ill-conditioned, generalized Tikhonov regularization ${ }^{8}$ has been used to solve this equation system. This corresponds to solving the following minimization problem:

$$
\hat{\mathrm{r}}=\underset{\mathrm{r}}{\arg \min }\left\{\|\mathrm{g}-\operatorname{Sr}\|_{2}^{2}+\alpha^{2} \sum\left(|\operatorname{Lr}|^{2}+\beta\right)^{\frac{k}{2}}\right\}
$$

where $\mathrm{g}, \mathrm{r}$ and $\mathrm{S}$ are the pulse-echo data, the unknown reflectivities and the forward model matrix, respectively. The first term in Eq.2 corresponds to the LMS solution to the linearized problem. The second term, which is included to stabilize the inverse problem, is the $l_{\mathrm{k}}$ norm $\left(\|\cdot\|_{\mathrm{k}}^{\mathrm{k}}\right)$ of a linear transformation of the unknown vector $r$ using a matrix operator $L$. $\beta$ is a small constant to allow the differentiation of the second term around zero for $\mathrm{k} \leq 1 . \alpha$ is known as the regularization parameter and controls the amount of regularization imposed on the solution. $\mathrm{L}$ was chosen to be the identity matrix because it is known to have filtering effects similar to TSVD 9 and is a natural way of regularizing an ill-posed problem. The performance of the reconstruction was quantified using the normalized mean square error (MSE), defined as:

$$
\mathrm{MSE}=100\|\hat{\mathrm{r}}-\overline{\mathrm{r}}\|_{2}^{2} /\|\overrightarrow{\mathrm{r}}\|_{2}^{2}
$$

where $\overline{\mathrm{r}}$ and $\hat{\mathrm{r}}$ are the true and reconstructed images respectively, both logarithmically compressed using a dynamic range of $40 \mathrm{~dB}$. 
Automatic parameter selection is an important aspect in regularization. Both general cross-validation (GCV) and L-curve ${ }^{10}$ were tested to select the best choice of the parameter $\alpha$ for fixed $\mathrm{k}$ and $\beta$.

To test the quality of the reconstructions, two-dimensional pulse-echo data were simulated using Field $\mathrm{II}^{11}$ corresponding to a single-element focused transducer with center frequency of $6 \mathrm{MHz}$ (wavelength $\lambda=250 \mu \mathrm{m}$ with a speed of sound of $1500 \mathrm{~m} / \mathrm{s}$ ) and focal distance of $19 \mathrm{~mm}$. Fractional bandwidths of $33 \%, 50 \%$ and $100 \%$, f/numbers of 1,2 and 3 , and distances between ROI and focus of $0,2.5$ and $5 \mathrm{~mm}$ were used for the simulations. The ROI used in the simulations is shown in Figure 1. It consists of two concentric regions, the inner one having a diameter of $1 \mathrm{~mm}$. The mean scatterer reflectivity in the outer region is 10 times larger than the one of the inner region. The scatterer density is 4 per $\lambda^{2}$. Because the largest bandwidth and smallest focal number used are $100 \%$ and 1 respectively, the minimum number of scatterers per resolution cell is roughly equal to 4 .

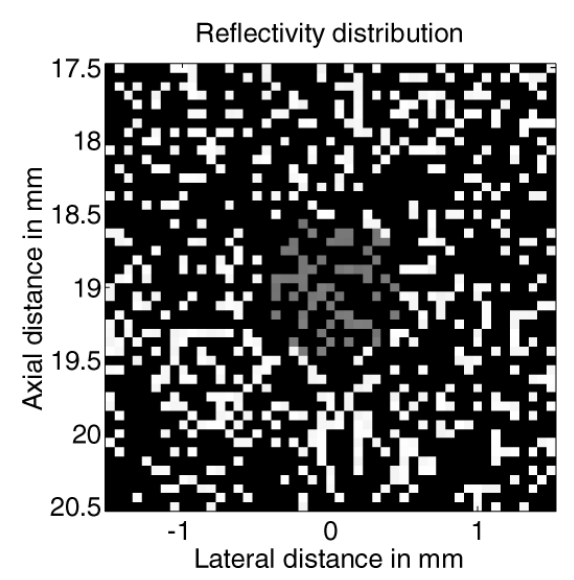

Figure 1. Reflectivity distribution used for the simulations.

\section{SIMULATIONS}

The simulations were performed by changing one physical parameter at a time among the bandwidth, the f/number and the distance to the focus, in order to analyze the individual effects of these parameters. For low SNR, the choice of $\mathrm{k}=2$ and $\mathrm{k}=0.5$ yields solutions that are oversmoothed or too sparse respectively. The choice of $\mathrm{k}=1$ represents a tradeoff between these two behaviors and hence the results corresponding to that particular choice 
will be shown here. With $\mathrm{k}=1$ the performance was somewhat insensitive to the choice of the parameter $\beta$, which was finally chosen to be $10^{-7}$. Both the L-curve and GCV yielded very similar results for this study, so only the GCV results are presented.

The MSE curves in Figure 2 indicate that among bandwidth, focal number and distance to focus, the bandwidth had the largest effect on the performance of the simulations. For an SNR of $20 \mathrm{~dB}$, the MSE corresponding to a bandwidth of $100 \%$ is close to $25 \%$, whereas for a bandwidth of $33 \%$ the error was roughly $86 \%$. For the same SNR, the error corresponding to changing the f/number to 3 is only $56 \%$ and the error corresponding to changing the distance between the focus and the ROI is only $35 \%$. The differences in changing the f/number from 2 to 3 or the distance between ROI and focus from 2.5 to $5 \mathrm{~mm}$ are not discernible from the MSE curves.
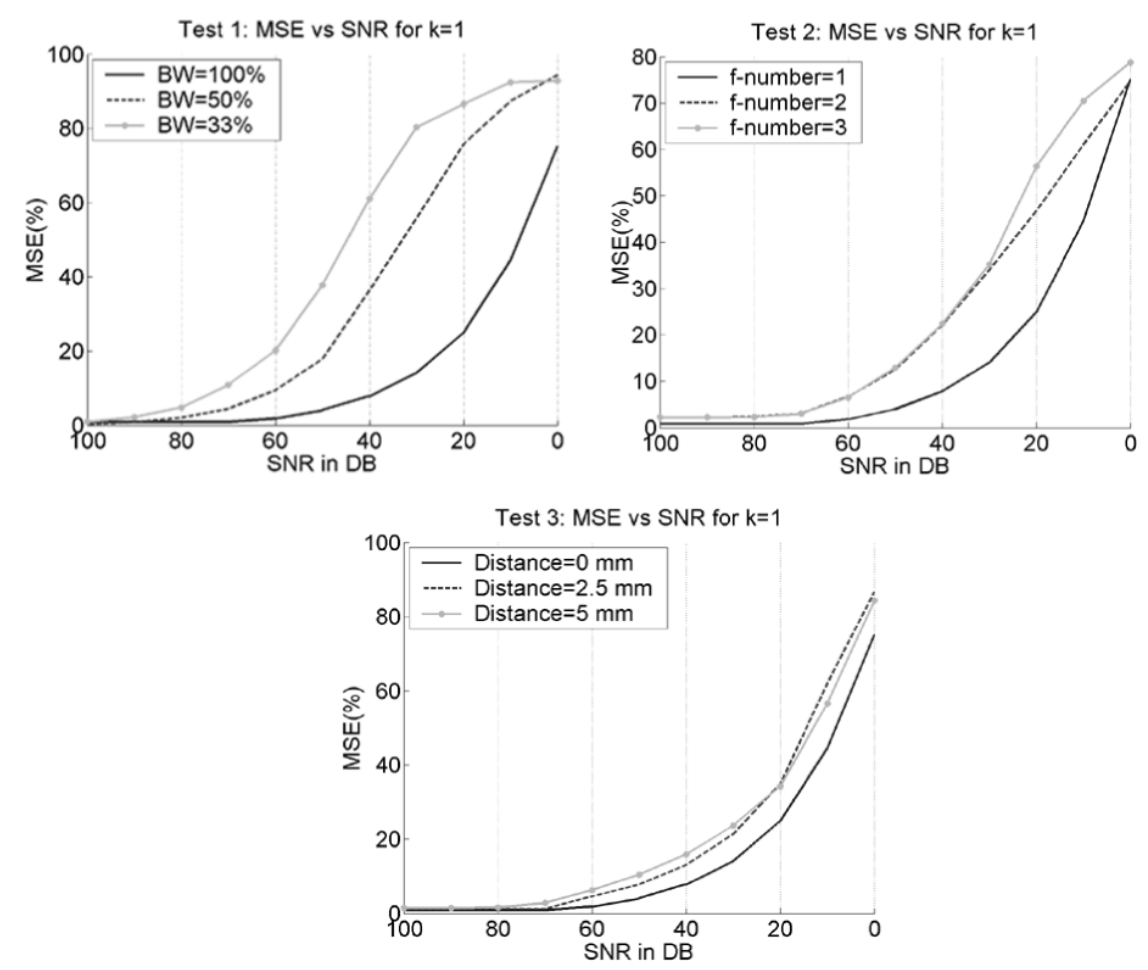

Figure 2. MSE curves for regularization reconstructions. Left top: effect of changing the bandwidth. Right top: effect of changing the f/number. Bottom: effect of changing the distance to the focus. 
Figure 3 shows the images corresponding to conventional techniques and regularization. The left column shows the B-mode (for the case of change in bandwidth and f/number) and SAFT (for the change of distance to the focus) images. The regularization reconstructed images selected using GCV are shown on the right column.

\section{CONCLUSIONS}

The performance of conventional imaging techniques such as B-mode and SAFT is mainly limited by the size of the resolution cell of the imaging system. This factor also has an impact on the performance of regularization techniques by determining the lower threshold for the SNR for which these approaches yield an image of better quality. A larger bandwidth, smaller f/number and smaller distance between ROI and focus result in better performance as measured by the MSE for fixed SNR. A lower bound of $20 \mathrm{~dB}$ for the SNR was found for this study. Below this threshold, contrast information and most of the image features were lost during the reconstruction process in order to control the distortion due to the noise. Further work will be required to improve the stability of the forward model and to use more appropriate a priori model information in order to use this technique in a more realistic scenario including the effects of modeling errors. 

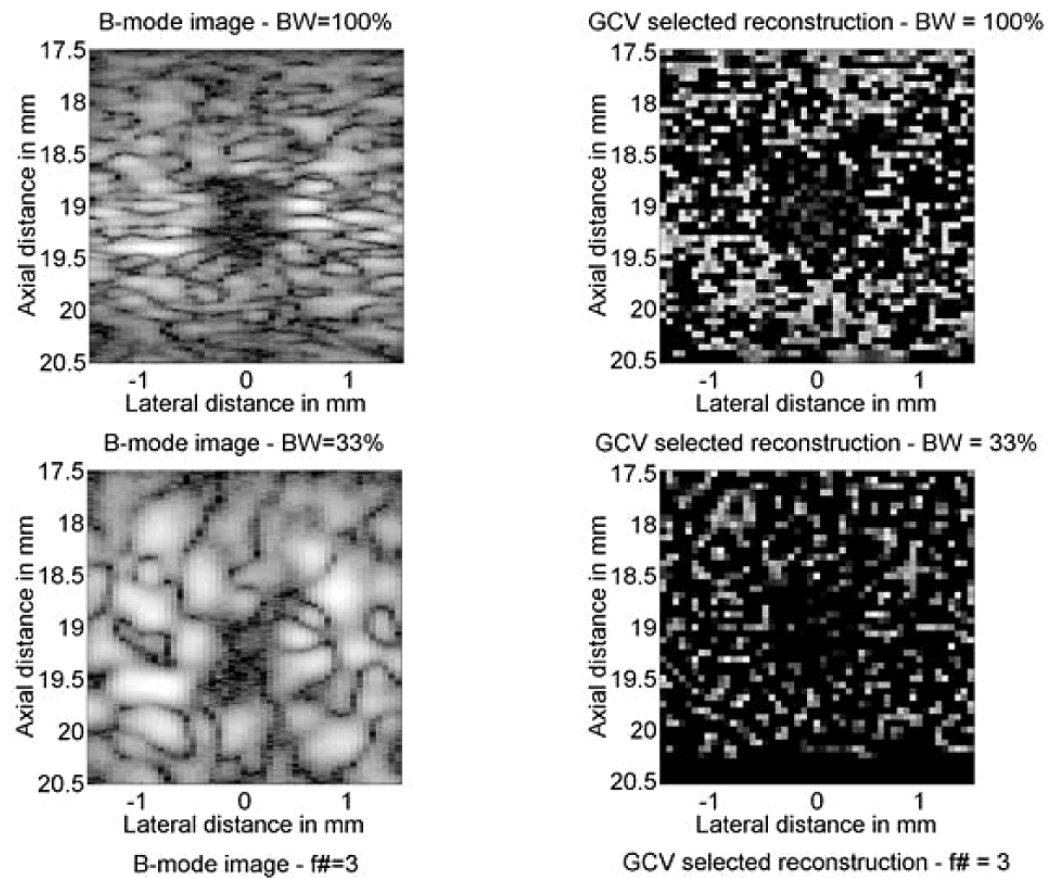

GCV selected reconstruction - BW $=33 \%$
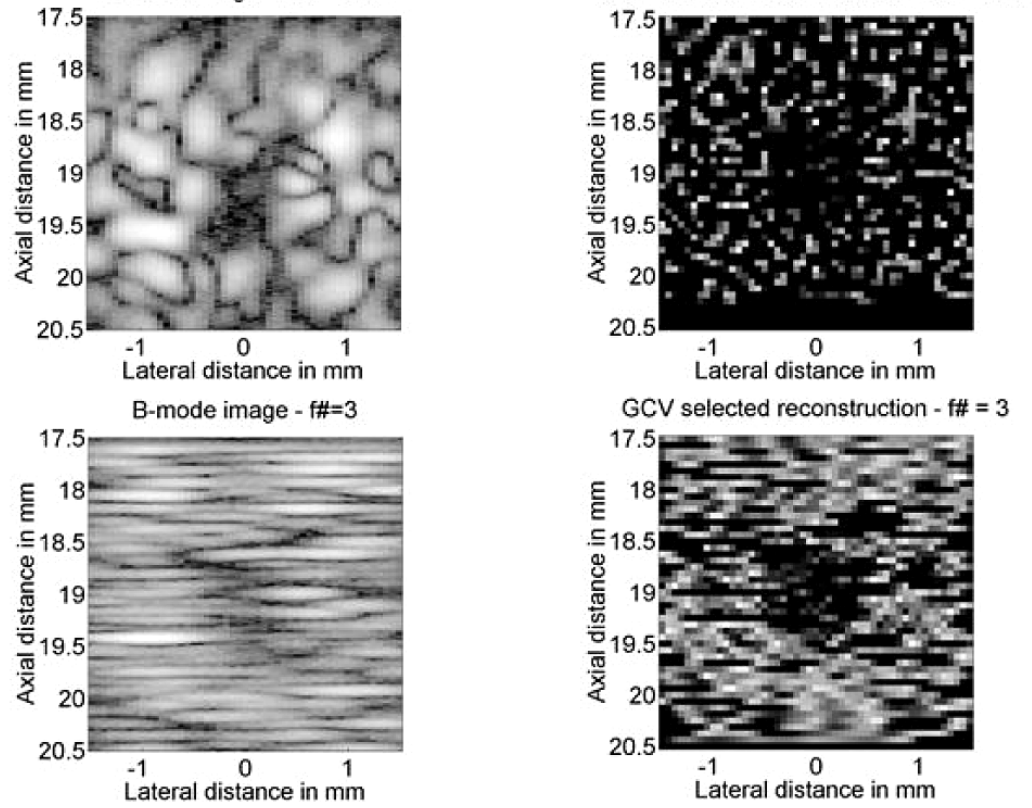

SAFT reconstruction with GCF - distance $=5 \mathrm{~mm}$

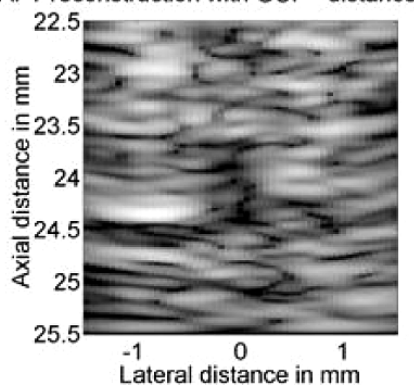

GCV selected reconstruction - distance $=5 \mathrm{~mm}$

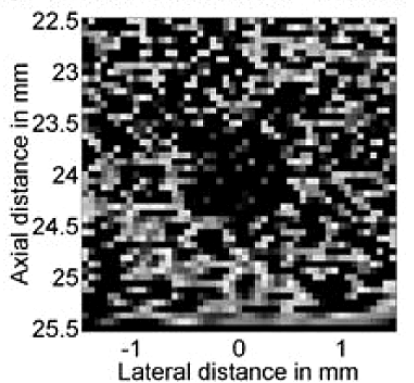

Figure 3. Reconstructed images for $\mathrm{SNR}=20 \mathrm{~dB}$. First row: $\mathrm{BW}=100 \%, \mathrm{f} / \#=1$ and distance to focus $=0 \mathrm{~mm}$. Second row: $\mathrm{BW}=33 \%, \mathrm{f} / \#=1$ and distance to focus $=0 \mathrm{~mm}$. Third row: $\mathrm{BW}=100 \%, \mathrm{f} / \#=3$ and distance to focus $=0 \mathrm{~mm}$. Fourth row: $\mathrm{BW}=100 \%, \mathrm{f} / \#=1$ and distance to focus $=5 \mathrm{~mm}$. 
Non-Quadratic Regularization with Speckle-Based Images

\section{REFERENCES}

1. Gordon S. Kino. Acoustic waves: devices, imaging, and analog signal processing. Prentice Hall, Inc (1997)

2. Christoph B. Burckhard. Speckle in ultrasound B-mode scans. IEEE Transactions on Sonics and Ultrasonics, 25(1), 1-6 (1978)

3. Fredrik Lingvall, Tomas Olofsson and Tadeusz Stepinski. Synthetic aperture imaging using sources with finite aperture: deconvolution of the spatial impulse response. Journal of the Acoustical Society of America, 114(1), 225-234 (2003)

4. Hesham Desoky, Abou Bakr M. Youssef and Yasser M. Kadah. Reconstruction using optimal spatially variant kernel for B-mode ultrasound imaging. SPIE Medical Imaging 2003: Ultrasonic Imaging and Signal Processing, 147-153 (2003)

5. Xiadong Zhang and Shira Broschat. A comparison of material classification techniques for ultrasound inverse imaging. Journal of the Acoustical Society of America, 111(1), 457-467 (2002)

6. Chao Liu, Yuanmei Wang and Pheng Ann Heng. A comparison of truncated total least squares with Tikhonov regularization in imaging by ultrasound inverse scattering. Physics in Medicine and Biology, 48(15), 2437-2451 (2003)

7. William C. Karl and Mujdat Cetin. Edge preserving image reconstruction for coherent imaging applications. IEEE International Conference on Image Processing, 481-484 (2002)

8. William C. Karl and Mujdat Cetin. Feature-enhanced synthetic aperture radar image formation based on non quadratic regularization. IEEE Transactions on Image Processing, 10(4), 623-631 (2001)

9. Heinz W. Engl, Martin Hanke and Andreas Neubauer. Regularization of inverse problems. Kluwer Academic Publishers (1996)

10. C. R. Vogel. Non-convergence of the L-curve regularization parameter selection method. Inverse Problems, 12(4), 535-547 (1996)

11. J.A. Jensen and N.B. Svendsen. Calculation of pressure fields from arbitrarily shaped, apodized, and excited ultrasound transducers. IEEE Transactions on Ultrasonics, Ferroelectrics and Frequency Control, 39(2), 262-267 (1992) 